\title{
Die Feinarbeit läuft
}

\section{Allgemein steigt die politische Bedeutung von Indikatoren. Zunehmend dienen sie als Informa- tions- und Kontrollinstrument hinsichtlich der Überprüfung der Erfüllung gesellschaftlicher und politischer Ziele. Ein internationales Beispiel ist die Entwicklung eines UNO-Systems zu Nach- haltigkeitsindikatoren. Die Ergebnisse der Testphase liefern einen wichtigen Beitrag für die deutsche Diskussion.}

W Von Uwe Taeger ie kann der Fortschritt in Richtung einer nachhaltigen Entwicklung bzw. der Umsetzung der Agenda 21 überprüft und damit ein Monitoring erreicht werden? Die UNKommission für nachhaltige Entwicklung (CSD) hat zu diesem Zweck 1995 ein Arbeitsprogramm zu Indikatoren für eine nachhaltige Entwicklung beschlossen, das vorerst noch bis April 2001 läuft. Kernelement war eine seit 1997 laufende internationale Testphase einer von der CSD vorgeschlagenen Indikatorenliste. In 22 Staaten, darunter auch Deutschland, wurden auf freiwilliger Basis 134 Indikatoren für alle Kapitel der Agenda 21 auf ihre praktische Umsetzbarkeit sowie auf ihre politische Relevanz und Aussagefähigkeit geprïft.

Das Bundesumweltministerium (BMU) war federführend für die Koordinierung der deutschen Testphase. Auf Grundlage der Konsultationen mit externen Wissenschaftlern und gesellschaftlichen Gruppen sowie den Beiträgen und Stellungnahmen der anderen Ressorts wurde ein Bericht der Bundesregierung erarbeitet, der im Dezember 1999 an die CSD übermittelt worden ist (1).

Hinsichtlich der Struktur sind die Kapitel der Agenda 21 den drei Säulen der nachhaltigen Entwicklung Soziales, Wirtschaft und Umwelt zugeordnet worden. Zusätzlich wurde die von der CSD vorgeschlagene Kategorie Institutionen aufgenommen. Das Indikatorensystem folgt einer Ordnungssystematik, die sich international wie national in den letzten Jahren zunehmend durchgesetzt hat - die Kategorisierung nach den Bereichen pressure bzw. driving force (Antrieb), state (Zustand) und response (Maßnahmen).

Dies gewährleistet die Anpassung an und den Vergleich mit anderen Indikatorensystemen zum Thema nachhaltige Entwicklung.

Im Ergebnis der deutschen Testphase hat sich gezeigt, dass viele der 134 Indikatorenvorschläge für ein Industrieland wie Deutschland nicht relevant oder nur wenig aussagekräftig waren. Der deutsche Testbericht fokussiert deshalb auf eine modifizierte Auswahl von Indikatoren, die den Problemen in Deutschland besser gerecht werden sollen. Ein Beispiel zur Illustration: Für das Agenda-Kapitel 40 Informationen für die Entscheidungsfindung wird von der CSD als institutioneller Zustandsindikator die Anzabl der Telefonanschlïsse pro 100 Einwohner vorgeschlagen. Der deutsche Bericht berücksichtigt diesen nicht, und schlägt stattdessen u.a. den Indikator Anzabl der Verbraucherzentralen vor. Der jetzt mit dem Bericht vorliegende nationale Satz umfasst insgesamt 218 Nachhaltigkeitsindikatoren.

Mit der Beteiligung wurden in Deutschland auch eine Reihe ergänzender Ziele verfolgt:

- Unterstïtzung der CSD-Initiative und Weiterentwicklung und Verbesserung des CSD-Konzepts,

- Förderung der nationalen Diskussion zu Entwicklung und Anwendung von Nachhaltigkeitsinschaftlichen Gruppen darüber.

Der deutsche Bericht wird damit gleichzeitig auch eine wichtige Grundlage für einen verbindlichen Satz von nationalen Nachhaltigkeitsindikatoren bilden, der im Rahmen der nationalen Nachhaltigkeitsstrategie zu entwickeln ist. Dabei wird es insbesondere darum gehen, die Anzahl der Indikatoren deutlich zu verringern, die Datengrundlagen zu prüfen, die Indikatoren hinsichtlich ihrer Aussagefähigkeit zu überprüfen sowie einen Abgleich mit den internationalen Arbeiten an Indikatorensystemen vorzunehmen.

Anmerkung

(1) BMU: Erprobung der CSD-Nachhaltigkeitsindikatoren in

\section{Der Autor}

Uwe Taeger ist Referent im Bundesministerium für Umwelt, Naturschutz und Reaktorsicherheit. Kontakt: BMU, Referat G 12, Alexanderplatz 6, 11055 Berlin. Tel. 01888-305-2451, Fax -3339, E-mail: taeger.uwe@bmu.de dikatoren, Förderung des Dialogs mit den gesell-
Deutschland. Bericht der Bundesregierung. Bonn 2000.

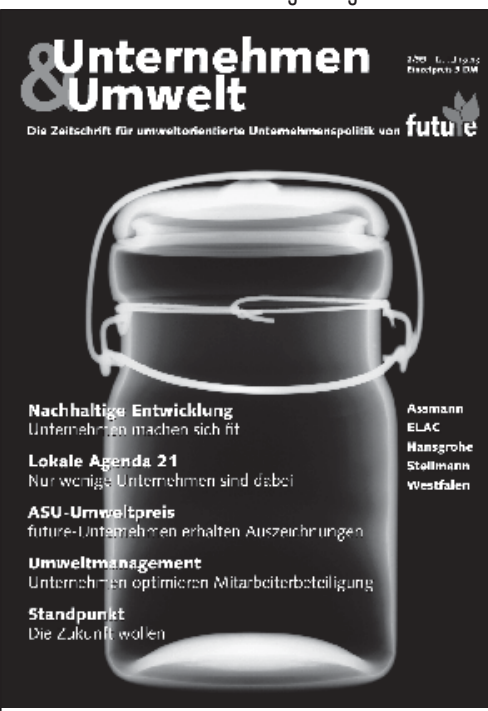

Unternehmen \& Umwelt

- ist die Zeitschrift für umweltorientierte Unternehmenspolitik von future e.V.,

- berichtet über den neuesten Stand zum betrieblichen Umweltmanagement in Theorie und Praxis,

- bietet vier Mal im Jahr ein Schwerpunktthema,

Unternehmensbeispiele, Literaturund Veranstaltungshinweise und aktuelle future-Nachrichten.

Zum Beispiel Heft 2/99:

Nachhaltigkeit

- Nachhaltige Entwicklung: Unternehmen machen sich fit.

- Lokale Agenda 21: Nur wenige Unternehmen sind dabei.

- Betrieblicher Umweltschutz: Assman, ELAC, Hansgrohe, Steilmann.

- Umweltmanagement: Unternehmen optimieren die Mitarbeiterbeteiligung.

Fordern Sie Ihr kostenloses Probeexemplar an: Fon: 0234 - 97995 - 13 Fax: $0234-97995-14$

future e.V. Büro Bochum Am Varenholt 123 44797 Bochum email: future@umis.de futule 
(c) 20I0 Authors; licensee IÖW and oekom verlag. This is an article distributed under the terms of the Creative Commons Attribution Non-Commercial No Derivates License (http://creativecommons.org/licenses/by-nc-nd/3.o/), which permits unrestricted use, distribution, and reproduction in any medium, provided the original work is properly cited. 